\title{
Capsule Commentary on Marshall et al., Effect of Patient Navigation on Breast Cancer Screening Among African American Medicare Beneficiaries: a Randomized Controlled Trial
}

\author{
Sara L. Jackson, M.D., M.P.H. \\ University of Washington, Seattle, WA, USA.

$\mathrm{E}$ xpanded coverage and access to health services does not guarantee that patients will utilize them, particularly in communities with a long-standing history of distrust of medical institutions, such as in Baltimore, MD. ${ }^{1}$ Black women are less likely to be screened for breast cancer and are diagnosed at later stages compared to white women, a gap that has been difficult to close for decades. ${ }^{2}$ Marshall et al. report a randomized community-based intervention using patient navigators among African American women in Baltimore that increased breast cancer screening compared to receipt of printed materials. ${ }^{3}$ These results add to the growing body of evidence supporting patient navigation as an effective tool for improving cancer screening rates among vulnerable populations. ${ }^{4}$

The study is strengthened by randomization of the intervention, which enables the authors to avoid pre- and postintervention comparisons that can be biased by temporal trends. The study's use of community-based partnerships and its focus upon Medicare-aged black women also advances the patient navigator evidence base. Though the mammography screening outcome was by self-report, claims data also demonstrated increased screening in the intervention relative to the control group.

As health care systems are asked to report quality measures for populations, new strategies that engage patients to improve health outcomes will be needed. In this study, differential loss to follow-up in the intervention group, particularly for those who were older, less educated, lower income, and with lower

health literacy, is consistent with adherence to other interventions. However, the dramatic increase in incident screening among women non-adherent to screening at baseline- $73.4 \%$ in intervention and $45.6 \%$ in control groups - suggests that focusing on this group non-adherent to screening is effective.

Limiting patient navigators to single disease states or interventions underutilizes the relationship that is built between navigator and patient. Integrating navigators into primary care has been useful, ${ }^{5}$ but is not common practice or reimbursed. Future study of health outcomes when navigators are embedded in primary care settings may demonstrate a diminished quality chasm, particularly if focused upon patients with cultural or environmental barriers to health care.

Conflict of Interest: The author has no conflicts of interest with this article.

Corresponding Author: Sara L. Jackson, M.D., M.P.H.; University of Washington, Seattle, WA 98104, USA (e-mail: sljack@uw.edu).

\section{REFERENCES}

1. Skloot R. The immortal life of Henrietta Lacks. New York: Crown Publishers; 2010. Print.

2. Silber JH, Rosenbaum PR, Clark AS, Giantonio BJ, Ross RN, Teng Y, et al. Characteristics associated with differences in survival among black and white women with breast cancer. JAMA. 2013;310(4):389-97.

3. Marshall JK, Mbah OM, Ford JG, Phelan-Emrick D, Ahmed S, Bone, L, et al. Effect of patient navigation on breast cancer screening among African American Medicare beneficiaries: a randomized controlled trial. J Gen Intern Med doi:10.1007/s11606-015-3484-2.

4. Percac-Lima S, Ashburner JM, Bond B, Os SA, Atlas SJ. Decreasing disparities in breast cancer screening in refugee women using culturally tailored patient navigation. J Gen Intern Med. 2013;28(11):1463-8.

5. Ferrante JM, Cohen DJ, Crosson JC. Translating the patient navigator approach to meet the needs of primary care. J Am Board Fam Med. 2010;23(6):736-44. 